\title{
Contractility of the Guinea Pig Bladder Measured In Situ and In Vitro
}

\author{
J. Groen, R. van Mastrigt, and R. Bosch \\ Department of Urology, Erasmus University, Rotterdam, The Netherlands
}

\begin{abstract}
To study the relative importance of neurogenic factors in detrusor contractility and to relate a total bladder in vitro contractility model to a previously described bladder wall strip model. active intravesical pressure values were compared in situ and in vitro in eight male guinea pigs. In situ. the active pressure was measured in spontaneous isometric and nonisometric micturition contractions. In vitro, the active pressure was measured in isometric contractions of the same bladders, developed in response to optimal electrical stimulation. The volume dependence of the active pressure generated by the bladder was measured in vitro in order to relate bladder capacity to the volume where the generated force is maximal and to determine the optimal volume at which to study detrusor contractility. The results indicated that in normal micturition the detrusor muscle was not fully stimulated: active pressure in isometric contractions in vivo was about $60 \%$ of the pressure values attained in vitro at the same bladder volume. Most micturitions occurred at a volume where the active pressure generated in vitro was about $80 \%$ of the maximal pressure. The active pressurebladder volume relationship complied with the sliding filament-cross bridge theory. In whole bladder preparations active stress was about twice as high as in strip;.

(1) $1 \% 94$ Wiley-L.tis. Inc
\end{abstract}

Key words: guinea pigs, bladder, contractility, urodynamics, micturition

\section{INTRODUCTION}

In a previous study we compared the contractility of guinea pig bladders in situ with the contractility of full thickness bladder wall strips taken from the same bladders [Groen et al., 1993]. The in situ contractions were non-isometric. As these contractions were evoked via a neurogenic pathway, contractility in situ was called neurogenically modulated. In vitro, contractility was evaluated from isometric contractions, induced by optimal electrical stimulation. Maximum active stress was taken as a measure of contractility. It was concluded that in a micturition contraction the bladder is not fully stimulated, i.e., contractility was smaller in the bladder in situ

Received for publication August 30, 1993; accepted May 6, 1994.

Address reprint requests to J. Groen, Department of Urology, Room EE 1630, Erasmus University Rotterdam. P.O. Box 1738, 3000 DR Rotterdam. The Netherlands.

Abbreviations: $V=$ bladder volume; $V_{1}=$ bladder tissue volume; $\sigma=$ maximum active stress in the bladder wall; $\mathrm{P}_{\mathrm{mc}}=$ maximum active intravesical pressure; $\mathrm{P}_{\mathrm{is}}=$ maximum extrapolated active intravesical pressure as derived from phase plot analysis; $C$ = time constant characterizing isometric pressure development as derived from phase plot analysis. 
than in the strips. This conclusion was based on the assumptions that the active stress generated by a longitudinal strip corresponds to the active stress generated by the complete bladder and that the intravesical pressure in non-isometric contractions is not significantly smaller than the pressure in isometric contractions.

In this study these assumptions are verified, and measurements are reported on total bladders in vitro under conditions of optimal electrical stimulation. The results are compared with the earlier described results on strips to assess the feasibility of the total bladder in vitro contractility model as opposed to the strip model. A comparison of maximum active pressures in isometric contractions in vivo and in vitro enabled the estimation of the intensity of the neurogenic stimulation in vivo, thereby providing our earlier conclusion with a quantitative basis.

In an in vivo study on guinea pig bladders, Uvelius and Gabella [1980] found that the active intravesical pressure, induced by pelvic nerve stimulation, was maximum at volumes which were small with respect to capacity. To verify the myogenic basis of this pattern, the active pressure-bladder volume relationship was determined in four bladders in vitro, thus also yielding the optimum volume at which to study bladder contractility. The resulting data were interpreted in terms of a force-length relationship in accordance with the sliding filament-cross bridge theory of contraction [Huxley, 1957].

\section{MATERIALS AND METHODS}

Eight male guinea pigs with a mean weight of $905 \mathrm{~g}$ (range: $710-1,145 \mathrm{~g}$ ) were used. Each animal was anaesthetized with urethane $(1.2 \mathrm{~g} / \mathrm{kg}$, i.p.). A small incision was made in the abdominal wall to localize the bladder, into which a $24 \mathrm{G}$ angiocatheter was inserted. Subsequently, the abdomen was closed again loosely with silk sutures. Body temperature was maintained by means of a heating pad.

A Harvard infusion pump was used to fill the bladder via the catheter with room temperature saline at a rate of $0.53 \mathrm{ml} / \mathrm{min}$. Pressure was measured via the same catheter using a disposable pressure transducer. The pressure signal was recorded on a strip chart recorder. Each bladder was filled 6 to 10 times, with intervals of 10 to 15 minutes [Maggi et al., 1987; Peterson et al., 1989]. In alternating filling cycles, the urethra was allowed to leak or occluded in the bulbar part with 3.5 metric silk suture. Thus two kinds of micturition contractions were measured: voiding contractions (when the urethra was allowed to leak) and isometric contractions (when it was occluded). The voided volume was collected and measured. Residual urine after voiding and following an isometric contraction was removed with a syringe attached to the catheter. For the voiding contractions, the residual volume was expressed as a percentage of the volume at the onset of micturition. The largest bladder volume in each animal at which a micturition contraction occurred was taken as its cystometric capacity. In each micturition contraction the maximum active pressure (defined as the maximum pressure during the contraction minus the pressure just before) was measured.

Maximum active stress $\sigma$ in the bladder wall due to the micturition contraction was calculated from the maximum active pressure $P_{m c}$, the bladder volume $V$ at the onset of micturition, and the tissue volume $V_{t}$ of the bladder as $\sigma=P_{m c} /\left(\left(1+V_{t} /\right.\right.$ $\left.\mathrm{V})^{2 / 3}-1\right)$. The derivation of this formula was described previously [Groen et al., 1993]. 
Following the in vivo experiments, the puncture site in the bladder induced by the catheter was closed by means of a 0.7 metric purse string suture. Both ureters were ligated. The bladder was excised and kept at room temperature in a buffer solution of the following composition: $\mathrm{NaCl} 137, \mathrm{KCl} 2.7, \mathrm{CaCl}_{2} 1.8, \mathrm{MgCl}_{2} 1.0, \mathrm{NaHCO}_{3} 8.0$, HEPES ( $\mathrm{N}$-[2-Hydroxyethyl]piperazine- $\mathrm{N}^{\prime}$-[2-ethanesulfonic acid]) 10.5 and glucose $5.1 \mathrm{mmol} / \mathrm{l} ; \mathrm{NaOH}$ was added to adjust the $\mathrm{pH}$ to 7.4 . After two $5 \mathrm{~F}$ catheters had been inserted via the remaining part of the urethra, the bladder was suspended in an organ bath containing $85 \mathrm{ml}$ of the same buffer solution, which was equilibrated with $95 \%$ $\mathrm{O}_{2} / 5 \% \mathrm{CO}_{2}$ at a temperature of $37^{\circ} \mathrm{C}$. The $\mathrm{pH}$ was in the range $7.3-7.4$ and the osmolality was close to $300 \mathrm{mosm}$. The solution could be refreshed within a few seconds from a heated and aerated storage container; this was done after every second stimulation. One catheter was connected to a Hospal infusion pump, the other one to a disposable pressure transducer. The bladder was filled to a volume corresponding to the mean of the previously infused volumes and allowed to accommodate for 1 hour before the onset of the experiments. Subsequently, the bladder was stimulated electrically for 4 seconds at intervals of 7 to 10 minutes by field stimulation, using two parallel platinum electrodes $(20 \times 30 \mathrm{~mm})$, one on each side of the bladder. In order to avoid electrolysis of the buffer solution, biphasic pulse stimulation was used. All isometric contractions were sampled at a rate of $10 \mathrm{~Hz}$ with a PDP 11 type computer and stored for further analysis. Phase plots, i.e., plots of the rate of change of pressure as a function of pressure, were made. From these the parameters $P_{i, o}$, the maximum extrapolated active pressure, and $\mathrm{C}$, the time constant for isometric pressure development, were derived [van Koeveringe and van Mastrigt, 1991].

To find optimum stimulus conditions, the pulse height, frequency and width were varied in four bladders, starting from $12.5 \mathrm{~V}, 100 \mathrm{~Hz}$, and $5 \mathrm{~ms}$, respectively, values based on the previously described measurements on strips. First, the applied voltage was semi-randomly varied between $5,7.5,10,12.5,15,20,25$, and $30 \mathrm{~V}$; the pulse frequency and width were kept constant. Subsequently, the frequency was varied between $25,50,100,200,400$, and $800 \mathrm{~Hz}$; the pulse height was kept at 12.5 $\mathrm{V}$. The pulse width was adapted to the frequency to attain a duty cycle of $50 \%$. Previous experiments have shown that the contraction force in pig urinary bladder muscle strips was maximum at this value [van Mastrigt and Glerum, 1985]. The $P_{\text {iso }}$ values found at the various parameter settings were normalized by dividing them by the value measured at $12.5 \mathrm{~V}, 100 \mathrm{~Hz}, 5 \mathrm{~ms}$. Measurements taken at the optimum parameter settings were used for further analysis.

In the remaining four bladders active pressure was measured at optimum parameter settings, while the bladder volume was varied. The active pressure-volume relationship was transformed into an active force-length relationship, taking the bladder inner circumference as the length of the bladder muscle and assuming a spherical shape [Uvelius and Gabella, 1980]. Force was calculated by multiplying the pressure by the area enclosed by the inner circumference.

Following the experiments the bladder was blotted and weighed. The volume was calculated, assuming a density of $1.0 \mathrm{~g} / \mathrm{ml}$. Maximum active stress values were calculated similarly as in vivo.

In each bladder, active pressure was thus measured under three different circumstances: in voiding contractions in vivo, in isometric contractions in vivo, and in isometric contractions in vitro under conditions of optimal electrical stimulation. The results were compared statistically using repeated measures analysis of variance and 
TABLE I. Bladder Weights of the Guinea Pigs Used and the Results of Cystometries for Both the Present Study and a Former Study*

\begin{tabular}{lcc} 
& $\begin{array}{c}\text { Present study } \\
(\mathrm{N}=8)\end{array}$ & $\begin{array}{c}\text { Former study } \\
(\mathrm{N}=7)\end{array}$ \\
\hline Bladder wet weight $(\mathrm{mg})$ & $572 \pm 34$ & $779 \pm 35$ \\
Cystometric capacity $(\mathrm{ml})$ & $4.1 \pm 0.3$ & $3.6 \pm 0.3$ \\
Residual volume $(\%)$ & $38 \pm 8$ & $48 \pm 5$ \\
$\mathrm{P}_{\text {Inc }}\left({\left.\mathrm{cm} \mathrm{H}_{2} \mathrm{O}\right)}^{\mathrm{O}\left(\mathrm{N} / \mathrm{cm}^{2}\right)}\right.$ & $19 \pm 1$ & $23 \pm 3$ \\
\hline
\end{tabular}

*The cystometric capacity of each animal was defined as the largest bladder volume at which a micturition reflex contraction occurred. The residual volume, given as a percentage of the volume at the moment micturition started, is the mean value of all voidings in each animal. $P_{m c}$ (the maximum active intravesical pressure) and $\sigma$ (the maximum active stress in the bladder wall) are the averaged results from the two voiding contractions with the highest active stress (in the former study $P_{m k}$ and $o$ were taken from threc contractions: in that study more voiding contractions were measured in each animal). Mean values and standard errors of the means are given.

the paired t-test. Taking into account a possible volume dependence of the measured pressure, only those results were used where all three pressure values in a bladder had been measured at approximately the same volume.

The results of the in vitro experiments were compared with the previously described results of in vitro experiments on bladder wall strips. To this end, the values of parameters measured on the strips were averaged for each bladder. The mean value and the standard error of the mean were calculated from these averaged values. Differences between the parameters determined on strips and on whole bladders were checked for significance using the unpaired two-tailed t-test.

\section{RESULTS}

The measured cystometrograms were similar to those described previously [Groen et al., 1993]. In Table I parameter values of both studies are compared.

During the first hour of in vitro measurement, the pressure generated by the bladders increased with repeated stimulations. Therefore, the parameters studied were taken from measurements after reproducible pressure values were obtained. Figure 1 shows the pulse height and pulse frequency dependence of $P_{i s o}$. It was concluded that $10 \mathrm{~V}, 200 \mathrm{~Hz}, 2.5 \mathrm{~ms}$, corresponded with optimum stimulus conditions.

In six bladders active pressure was measured under the three different circumstances (voiding contractions in vivo, isometric contractions in vivo, isometric contractions in vitro) at similar volumes. Table II shows that the pressure values were significantly different, while the corresponding volumes were not.

Table III shows the results of the in vitro measurements, taken at volumes similar to the in vivo volumes. It also shows the results from the previously published measurements on bladder wall strips. It can be seen that in the whole bladder preparations maximum active stress exceeded the stress in the strips by almost exactly a factor of 2 . 


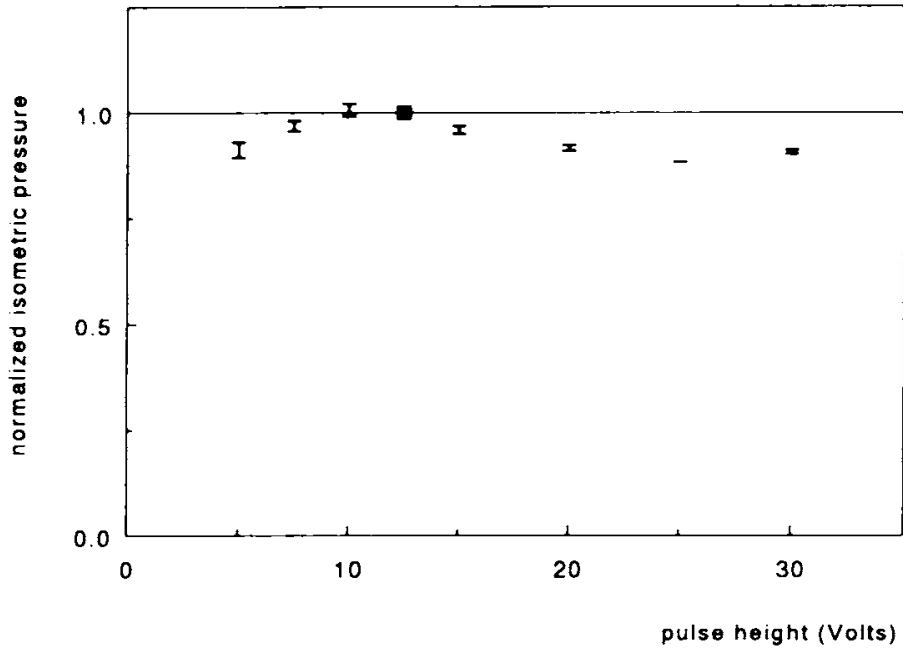

A

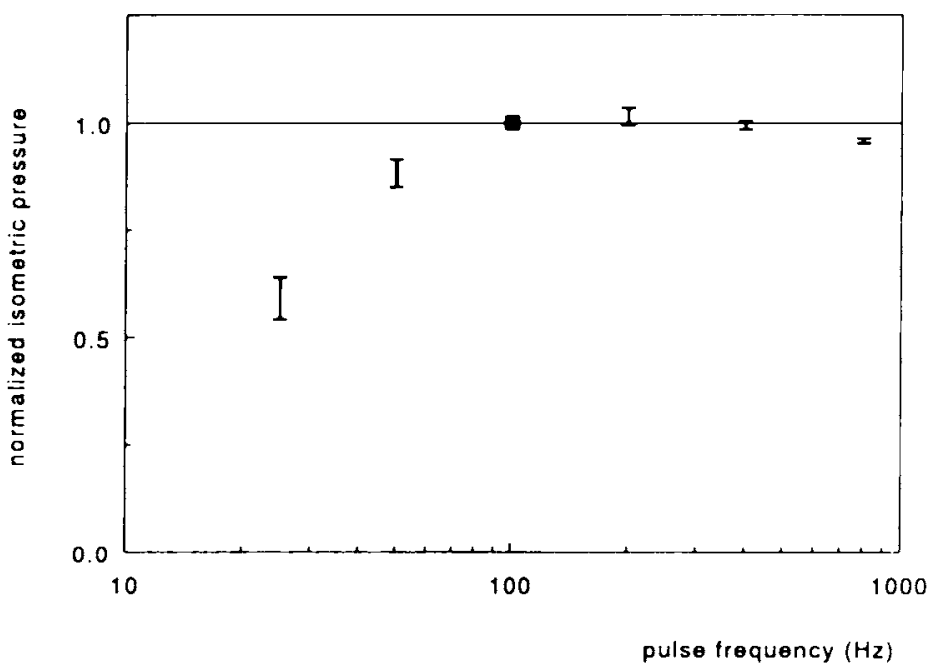

B

Fig. 1. Optimization of parameter settings for electrical stimulation of total guinea pig bladders in vitro. Averaged results of 4 bladders and the corresponding standard errors of the mean. A: Pulse height dependence of extrapolated active pressure. The pressures were normalized to the value at $12.5 \mathrm{~V}(\mathbf{D})$. Pulse frequency was $100 \mathrm{~Hz}$; pulse width was $5 \mathrm{~ms}$ (duty cycle $50 \%$ ). Biphasic pulses were used. B: Pulse frequency dependence of extrapolated active pressure. The pressures were normalized to the value at 100 $\mathrm{Hz}$ (D). Pulse height was $12.5 \mathrm{~V}$; duty cycle was $50 \%$.

The active pressure, generated by a bladder upon electrical stimulation, was strongly dependent on its volume (Fig. 2A). The force-length relationship derived from this data (Fig. 2B) shows that active force was maximally $1.60 \mathrm{~N}$ at a bladder circumference of approximately $6.5 \mathrm{~cm}$. This corresponds to a volume of $4.6 \mathrm{ml}$, 
TABLE II. Active Pressures at the Shown Bladder Volumes, Measured in Six Bladders Under Three Different Circumstances: In Voiding Contractions In Vivo, In Isometric Contractions In Vivo, and In Isometric Contractions In Vitro Under Conditions of Optimum Electrical Stimulation*

\begin{tabular}{lcccc}
\hline & $\begin{array}{c}\text { Voiding } \\
\text { contraction } \\
\text { in vivo }\end{array}$ & $\begin{array}{c}\text { Isometric } \\
\text { contraction } \\
\text { in vivo }\end{array}$ & $\begin{array}{c}\text { Isometric } \\
\text { contraction } \\
\text { in vitro }\end{array}$ & $P$ \\
\hline Active pressure $\left(\mathrm{cm} \mathrm{H}_{2} \mathrm{O}\right)$ & $19.5 \pm 1.0$ & $39.3 \pm 2.8$ & $63.2 \pm 1.8$ & $<0.0005$ \\
Bladder volume $(\mathrm{mL})$ & $2.5 \pm 0.3$ & $2.8 \pm 0.3$ & $2.8 \pm 0.2$ & 0.53 \\
\hline
\end{tabular}

* Mean values and standard errors of the means are given. The $P$ values resulted from repeated measures analysis of variance. Pairwise application of the t-test showed that all three pressure values were significantly different from each other $(P \leq 0.001)$.

TABLE III. Results of Electrical Stimulation Experiments on Eight Guinea Pig Bladders In Vitro Compared With Previously Described Results on Strips From Eight Other Bladders*

\begin{tabular}{|c|c|c|c|}
\hline & Whole bladders & Bladder wall strips & $P$ \\
\hline$V(\mathrm{ml})$ & $2.8 \pm 0.2$ & $3.2 \pm 0.2$ & 0.27 \\
\hline$P_{1 W}\left(\mathrm{~cm} \mathrm{H}_{2} \mathrm{O}\right)$ & $63.0 \pm 1.7$ & - & \\
\hline$C(s)$ & $0.91 \pm 0.09$ & $0.85 \pm 0.04$ & 0.61 \\
\hline$\left(\mathrm{N} / \mathrm{cm}^{2}\right)$ & $5.03 \pm 0.35$ & $2.56 \pm 0.30$ & $<0.0005$ \\
\hline
\end{tabular}

*In the whole bladder preparations $V$ is the bladder volume; in the strips it is the volume corresponding with the stretched length of the strips. (In these experiments the in situ bladder was filled to the volume $V$ and strips were marked; in vitro the strips were stretched to the marked length.) $P_{\mathrm{ss}}$ and $\mathrm{C}$ are the maximum extrapolated pressure and the time constant for isometric pressure or force development as derived from phase plots. The maximum active stress is denoted by $\sigma$. Mean values and standard errors of the means are given; the $P$ values resulted from the unpaired two-tailed t-test.

which is considerably larger than the volume of 2.5 to $2.8 \mathrm{ml}$ at which most micturition contractions in vivo occurred (Table II).

\section{DISCUSSION}

Micturition reflex contractions were studied under two different circumstances: with an unmanipulated urethra and with an occluded urethra. In the first situation the bladder emptied; these micturition contractions were called voiding contractions. When the urethra was occluded, the contractions were called isometric. As in these contractions no shortening of the detrusor muscle took place, higher active pressure values were expected in accordance with the force-velocity relation. Table II shows that the difference in active pressure in voiding contractions and in isometric contractions was indeed quite large. This result is in agreement with the observations of Peterson et al. [1989]: also in urethane-anaesthetized guinea pigs, these investigators measured active pressure values of $22 \pm 4$ and $39 \pm 3 \mathrm{~cm} \mathrm{H} \mathrm{H}_{2} \mathrm{O}$ in voiding contractions and in isometric contractions. 


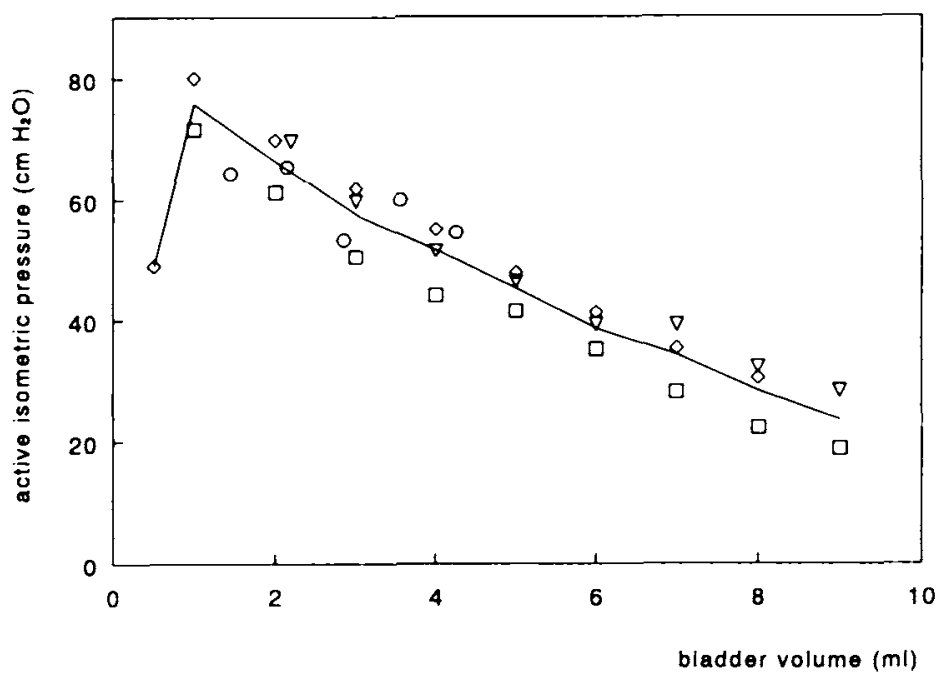

A

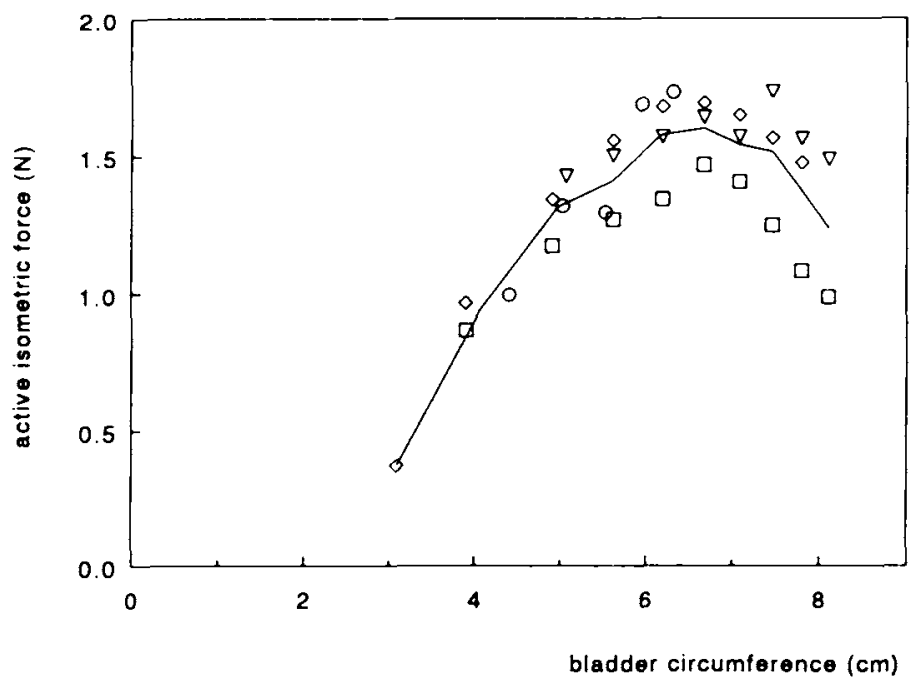

B

Fig. 2. A: Bladder volume dependence of the extrapolated active isometric pressure, measured in vitro under conditions of optimal electrical stimulation. Results of four bladders, represented by four different symbols, $(\diamond, \square, \bigcirc, \nabla)$. B: The relationship between active force and bladder circumference, calculated from the data in (A). The solid lines connect the mean values of clusters of data.

As described in the introduction, maximum active pressure attained during isometric contractions in vivo was considered a measure of the neurogenically modulated contractility of the detrusor muscle, i.e., it is influenced by both neurogenic and myogenic factors. It can be directly compared with the maximum active pressure developed in vitro under conditions of optimal stimulation. In this study, electrical stimulation was used. With optimum parameters electrically induced contractile re- 
sponses are comparable in magnitude with potassium chloride induced responses [van Koeveringe and van Mastrigt, 1991] and thus can be considered to represent maximal contractility. The stimulus optimization curves (Fig. 1) and optimum stimulus parameters were similar to those found for the strips. Tests with tetrodotoxin were not done in the present study because it was our aim to measure maximal contractility irrespective of the stimulatory pathway involved.

Table II confirms our carlier conclusion that in a micturition contraction the neurogenically modulated contractility of the detrusor muscle is smaller than its maximal contractility, i.e., in micturition the bladder is not fully stimulated. In vitro studies by Levin et al. [1991] showed that complete bladder emptying can occur at stimulation intensities at which the generated pressure is submaximal. This phenomenon was interpreted as a "reserve capacity to empty."

In our earlier study on full thickness bladder wall strips, maximum active stress was taken as the measure of contractility. The difference between the in vivo and in vitro contractility was less convincing than in the present study. Therefore, it is of importance to compare the measurements on strips with those on whole bladder preparations. This was previously done by Levin et al. [1991]. Using variable stimulation intensities, these authors showed that the contractile response of bladder strips was proportional to the contractile response of whole bladder preparations. When comparing the maximum active stress measured in both preparations, the structure of the muscle wall of the bladder should be kept in mind. It is considered to consist of three ill-defined layers, an outer and an inner longitudinal layer and an intermediate circular layer, which is usually the thickest [Uvelius, 1980]. Each layer seems to contain a number of muscle bundles. As the direction of these bundles is not uniform, the adjectives "Iongitudinal" and "circular" only express their general direction in the respective layers. In whole bladder preparations all bundles of the detrusor muscle are involved in the development of force (pressure), while in strips only the longitudinal bundles are involved; the contraction of the bundles cut transversally is not recorded by the force transducers. If all three layers were equally thick and all muscle bundles ran in the longitudinal and circular direction perfectly, a ratio of the maximum active stress in whole bladders and in strips of 3:2 would be expected. In the other extreme situation, where in each muscle layer all bundles run in randomly distributed directions, a ratio of $2: 1$ is expected. The results shown in Table III fit in with both situations described.

The in vitro measured active pressure decreased almost linearly with increasing volume after an initial maximum had been reached (Fig. 2A). Although in this case the maximum is based on one measurement only, similar relationships were found by Uvelius and Gabella [1980] in in vivo measurements on the guinea pig and by Kaplan et al. [1991] in in vitro rabbit bladders. The force-length relationship was calculated from the data in Figure 2A. The results (Fig. 2B) were in agreement with those reported for pig bladder strips [Griffiths et al., 1979; van Mastrigt and Glerum, 1985; van Mastrigt, 1988] and for strips from the guinea pig bladder [Longhurst et al., 1990] (although in the latter case the decrease of force beyond the optimal length was reported not to be significant). This force-length relationship, and therefore the pressure-volume relationship, is as predicted by the sliding filament-cross bridge theory [Huxley, 1957].

In the present study micturition contractions occurred at volumes well below the volume at which the active force in vitro was maximal, i.e., at volumes correspond- 
ing to the rising part of the force/length relationship (Fig. 2). As a result of the fact that forces rise steeply with bladder circumference in this part of the force/length characteristic, the decrease of isometric pressure with bladder volume, caused by geometrical factors, is only moderate at mean capacity. This enables the bladder to empty over a wide range of volumes. At mean capacity, active isometric pressure in vitro was approximately $80 \%$ of maximum.

\section{CONCLUSIONS}

During micturition the guinea pig detrusor muscle is not fully stimulated. In our data the isometric pressure during a micturition contraction amounted on average to $60 \%$ of the pressure attained during electrical stimulation of the same bladder in vitro at the same volume. In addition to that, micturition usually occurred at a volume where the pressure developed by the detrusor in vitro was about $80 \%$ of its maximum value. The in vitro pressure is volume dependent and complies with the sliding filament-cross bridge theory. At optimal electrical stimulation, the stress induced in the wall of a whole bladder preparation is about twice as high as the stress induced in a longitudinal full thickness bladder wall strip. This difference can be understood from the structure of the bladder wall.

\section{REFERENCES}

Griffiths DJ, van Mastrigt R, van Duyl WA, Coolsaet BLRA (1979): Active mechanical properties of the smooth muscle of the urinary bladder. Med Biol Eng Comput 17:281-290.

Groen J, van Asselt E, van Mastrigt R, Bosch R, van Koeveringe GA (1993): Comparison of detrusor contractility of guinea pig bladders in situ and strips from these in vitro. J Urol 150:1002-1006

Huxley AF (1957): Muscle structure and theories of contraction. Prog Biophys Biophys Chem 7:255318.

Kaplan SA, Blaivas JG, Brown WC. Levin RM (1991): Parameters of detrusor contractility: I: The effects of electrical stimulation, hysteresis, and bladder volume in an in vitro whole rabbit bladder model. Neurourol Urodyn 10:53-59.

Levin RM, Kato K. Lin AT, Whitmore K, Wein AJ (1991): Correlation of in vitro pressure generation with urinary bladder function. Neurourol Urodyn 10:185-192.

Longhurst PA. Kang J. Wein JA. Levin RM (1990): Comparative length-tension relationship of urinary bladder strips from hamsters, rats, guinea pigs, rabbits and cats. Comp Biochem Physiol 96A:221-225.

Maggi CA. Meli A. Santicioli P (1987): Neureffector mechanisms in the voiding cycle of the guinea-pig urinary bladder. J Auton Pharmacol 7:295-308.

Peterson JS. Hanson RC. Noronha-Blob L (1989): In vivo cystometrogram studies in urethane-anesthetized and conscious guinea pigs. J Pharmacol Methods $21: 231-241$.

Uvelius B (1980): Relation between mechanical and morphological characteristics in urinary bladder smooth muscle. Acta Physiol Scand (Suppl) 483:5-51.

Uvelius B. Gabella G (1980): Relation between cell length and force production in urinary bladder smooth muscle. Acta Physiol Scand 110:357-365.

van Koeveringe GA, van Mastrigt $R$ (1991): Excitatory pathways in smooth muscle investigated by phase-plot analysis of isometric force development. Am J Physiol 261:R 138-R 144

van Mastrigt $R$ (1988): The length dependence of the series elasticity of pig bladder smooth muscle. J Muscle Res Cell Motil 9:525-532

van Mastrigt R, Glerum JJ (1985): Electrical stimulation of smooth muscle strips from the urinary bladder of the pig. J Biomed Eng 7:2-8. 